\title{
Televisión sin fines de lucro en la Argentina de la Ley Audiovisual: el caso de Barricada TV
}

\section{Resumen}

\author{
Santiago Marino \\ Universidad Nacional de Quilmes \\ sgomarino@gmail.com
}

\author{
Josep Àngel Guimerà Orts \\ Universitat Autònoma de Barcelona \\ josepangel.guimera@uab.cat
}

La aprobación de la Ley de Servicios de Comunicación Audiovisual (LSCA) en la Argentina en 2009 abrió la puerta a una modificación a fondo del sistema radiotelevisivo del país. Entre otros motivos, porque otorgaba una clara importancia a los medios sin fines de lucro y comunitarios, a los cuales reservaba un tercio del total del espacio radioeléctrico. De esta manera, unos actores perseguidos hasta 2009 pasaban a protagonizar (potencialmente) un rol central en el nuevo escenario mediático que se quería construir. Pero a finales de 2015, la situación dista mucho de la prevista en la ley en lo referido a las televisiones comunitarias y sin afán de lucro. El presente trabajo tiene dos objetivos. En primer lugar, describir e interpretar la aplicación de la LSCA entre 2009 y 2015 en los aspectos relativos a la televisión sin fines lucrativos y comunitaria. Las investigaciones previas apuntaron hacia una aplicación escasa y sesgada de estos aspectos debido a otras prioridades del gobierno. Esto se confirma para 2015 y se añaden dudas sobre el sector después de que el nuevo ejecutivo surgido de las elecciones de ese mismo año haya modificado la LSCA hasta desvirtuarla. En segundo lugar, generar conocimiento original sobre las relaciones entre Estado y medios comunitarios, un aspecto poco estudiado. Se aborda cómo se ha pasado de la persecución a la legalización y como esta resulta ambivalente para unos actores históricamente recelosos del Estado: si bien obtienen seguridad jurídica y recursos económicos vía subvenciones, también constatan la necesidad de cumplir una ley exigente y un mayor control y seguimiento de la administración. La metodología seguida ha sido cualitativa, basada en el análisis documental tanto de investigaciones previas como de documentación oficial y de prensa argentina. Esto se ha completado con entrevistas en profundidad a actores implicados en el proceso estudiado.

Palabras clave: políticas de comunicación, televisión comunitaria, Ley de Servicios de Comunicación Audiovisual, Argentina. 


\title{
Nonprofit television in Argentina after the Audiovisual Law: the Barricada TV case
}

\begin{abstract}
With the passing of Argentina's Audiovisual Communication Services Law (LSCA) in 2009, the country's radio and TV media system went through a profound transformation. Among its many features, the law paid special attention to nonprofit community media, to which it reserved a third of the radio spectrum. Media actors who had been persecuted up to 2009 came to (potentially) occupy a central role in the new media landscape that was being built. However, towards the end of 2015, the condition of nonprofit community television differed markedly from what had been outlined in the law. This paper has two objectives. First, it seeks to describe and interpret LSCA's application between 2009 and 2015 in matters concerning nonprofit community television. Previous investigations have found the law's application to be lackluster and biased due to the government having other priorities. This situation remained the same in 2015, and was aggravated when the new executive, voted into office that year, modified the LSCA to the point of devaluing it. Second, it hopes to deepen our understanding of the relationship between the state and community media, a subject that has received little attention. In what follows, the paper will address how nonprofit community television in Argentina has made the transition from persecution to legalization, and how this shift has had uncertain results for media actors who have been historically suspicious of the state. Although they have gained legal protection and economic resources through subsidies, they must now abide by a rigorous law as well as greater administrative control and monitoring. We have followed a qualitative methodology, basing our research on the analysis of both previous investigations and documentation from official and journalistic sources. We have also supplemented these materials with in-depth interviews with media actors involved in this process.
\end{abstract}

Keywords: communication policies, community television, Audiovisual Communication Services Law, Argentina.

\section{Televisão sem fins lucrativos na Argentina da Lei Audiovisual: o caso de Barricada TV}

\section{Resumo}

A aprovação da Lei de Serviços de Comunicação Audiovisual (LSCA) na Argentina no ano 2009 abriu a porta a uma modificação profunda do sistema radiotelevisivo do país. Entre outros motivos, porque outorgava uma clara importância aos meios comunitários e sem fins lucrativos, aos quais reservava um terço do total do espaço radioelétrico. Desse jeito, os atores perseguidos até 2009 passaram a protagonizar (potencialmente) um rol central no novo cenário midiático que se queria construir. Mas, no final do ano 2015, a situação estava longe da prevista na lei no referido às televisões comunitárias e sem fins de lucro. O presente trabalho tem dois objetivos. Em primeiro lugar, descrever e interpretar a aplicação da LSCA entre 2009 e 2015 nos aspetos relativos à televisão comunitária e sem fins lucrativos. Pesquisas prévias apontavam para uma aplicação escassa e distorcida destes aspectos devido a outras prioridades do governo. Isso foi confirmado para 2015 e foram adicionadas duvidas sobre o setor depois de que o novo executivo surgido das eleições desse mesmo ano tenha modificado a LSCA até desvirtuar ela. Em segundo lugar, gerar conhecimento original sobre as relações entre Estado e meios comunitários, um aspecto pouco estudado. Aborda-se como passaram da persecução a legalização, e como essa legalização resulta ambivalente para uns atores historicamente receosos do Estado: embora eles obtém garantias jurídicas e recursos económicos através de subvenciones, também constatam a necessidade de 
cumprir uma lei exigente e um maior controle e seguimento da administração. A metodologia seguida tem sido qualitativa, baseada no analise documental tanto de pesquisas prévias quanto de documentação oficial e de imprensa argentina. Isso tem se completado com entrevistas em profundidade realizadas a atores implicados no processo estudado.

Palavras chave: políticas de comunicação, televisão comunitária, Lei de Serviços de Comunicação Audiovisual, Argentina.

\section{Introducción}

La aprobación de la Ley 26522 de Servicios de la Comunicación Audiovisual (LSCA) en 2009 supuso un antes y un después para el sector radiotelevisivo argentino. La nueva norma dotaba al país de una ley democrática al derogar el Decreto-Ley $22285 / 80$, elaborado por la dictadura militar, e imprimía un giro muy importante en la concepción del papel de los medios en el país.

El decreto de la dictadura, reformado en múltiples ocasiones en la "larga década neoliberal 1989-2001" (Marino, 2013), configuraba un sector fuertemente centralizado en Buenos Aires y mayoritariamente en manos privadas y con intereses comerciales, sin lugar para los medios no lucrativos - que tenían impedido el acceso al espacio radioeléctrico- y sin organismos de control independientes del gobierno (Postolski \& Marino, 2009, p. 171). En cambio, la LSCA limitaba la concentración y a la propiedad cruzada, establecía requerimientos de transparencia a los licenciatarios, imponía obligaciones de servicio público a los medios privados comerciales, creaba una autoridad regulatoria independiente - la Autoridad Federal de Servicios de Comunicación Audiovisual, AFSCA - y reservaba un tercio de las todas las frecuencias para organizaciones sin ánimo de lucro. La nueva ley - fuertemente debatida y con abundante participación ciudadana en su elaboración- había recibido el reconocimiento de la ONU, que la consideró una norma ejemplar a nivel internacional no solo por su contenido sino también por el proceso de elaboración, tan abierto y participado (Becerra, Marino \& Mastrini, 2012, p. 6-7; Marino, 2016).

Marino, Mastrini y Becerra (2011, p. 12) destacan como uno de los aspectos más novedosos de la nueva ley "la reserva del 33\% de todo el espectro para el sector privado no comercial", hecho que califican de "inédito en el mundo". En este aspecto, en concreto la ley suponía un giro copernicano: con el decreto-ley de la dictadura, estos medios estaban expresamente excluidos del éter y habían sido perseguidos durante casi toda la democracia (Marino, 2009). Ahora bien, diversas investigaciones apuntan a que los aspectos de la ley relativos a los medios no lucrativos no fueron implantados o lo fueron de manera sesgada e incompleta en algunos casos, dando lugar a una situación de falta de seguridad jurídica de estos actores hasta finales de 2015 (Guimerà, 2013; Marino, 2016; Segura, 2013; Segura \& Weckesser, 2016). 
En 2016, un cambio en la presidencia de la República Argentina ha supuesto otro cambio en la política de medios del país. Como sostiene Becerra (2016b), la llegada al poder de Mauricio Macri ha supuesto un proceso restaurador en el que se vuelve a definir el sistema de medios a partir de los mismos principios privatistas y comerciales que establecía el decreto de la dictadura. Mediante el Decreto de Necesidad y Urgencia (DNU) 267/15, el gobierno de Macri modificó aspectos centrales de la LSCA al eliminar los límites a la propiedad cruzada y la concentración de medios; y subordinar la autoridad reguladora al poder ejecutivo, derogó el AFSCA y creó el Ente Nacional de Comunicaciones (ENaCom), cuyos directores puede nombrar libremente el Poder Ejecutivo. Estas decisiones se tomaron en el marco de un proceso de reforma del marco legal relativo al audiovisual y las telecomunicaciones en el que, de momento, no se han visto afectados de manera directa los medios sin fines de lucro ni, en especial, la reserva de un tercio del espacio radioeléctrico (Becerra, 2016a; Marino, 2016). De hecho, en agosto de 2016 las organizaciones que representan al sector, organizadas en el colectivo Interredes ${ }^{1}$, fueron notificadas por el ENaCom de que el Programa de Fondos Concursables FOMECAS tenía nuevo reglamento y se realizaría en el primer año de la actual gestión. Pero al cierre de este trabajo no fue publicado en resolución alguna. Y no hay novedades sobre las deudas de los fondos correspondientes a 2015. Todo lo apuntado hasta ahora convierte los años 2009 a 2015 en un periodo históricamente delimitado dentro de las políticas de comunicación en el país.

El presente trabajo presenta dos objetivos. Por una parte, describir e interpretar la aplicación de la LSCA entre 2009 y 2015 en los aspectos relativos a los medios sin afán de lucro, uno de sus elementos centrales. Investigaciones previas (Guimerà, 2013; Marino, 2016; Segura, 2013) apuntaban hacia una aplicación escasa y sesgada de estos aspectos debido a otras prioridades del gobierno presidido por Cristina Fernández de Kirchner. Así, solo a finales de 2015 y ya con la amenaza de un cambio de gobierno en ciernes, se concedieron frecuencias a un pequeño grupo de medios no comerciales. Hasta 2013, estos medios fueron notablemente ignorados tanto por el ejecutivo como por la AFSCA, que en ese año otorgó autorizaciones provisionales de emisión ante su incapacidad de abrir un concurso de concesiones. El segundo objetivo es generar conocimiento original sobre un aspecto poco estudiado: las relaciones entre Estado y medios comunitarios en un momento histórico en que estos son expresamente reconocidos por la ley. Se quiere saber cómo han sido estas relaciones en los seis años de gestión kirchnerista de la LSCA, si estas han evolucionado en el tiempo y en qué sentido lo hicieron. Se presta especial atención a la concepción de que los medios no lucrativos tienen del Estado después de la plena legalización de 2015 tras décadas de desconfianza, persecuciones y menosprecios.

\footnotetext{
http://amarcargentina.org/tag/interredes/
} 
De acuerdo con las características del objeto de estudio y de los objetivos definidos, para llevar a cabo esta investigación se ha optado por el análisis de caso. Como apunta Yin (2009, p. 18), se trata de una estrategia de investigación que permite "investigar un fenómeno contemporáneo en su contexto de vida real, especialmente cuando las fronteras entre el fenómeno y el contexto no son claramente evidentes". Es decir, se usa el caso de estudio cuando se quiere deliberadamente "cubrir las condiciones contextuales creyendo que estas deben ser altamente pertinentes" para el estudio escogido. Desde nuestro punto de vista, estudios previos apuntan a una clara relación entre contexto político - gobiernos kirchneristas- y el fenómeno estudiado: las políticas sobre medios comunitarios y sus eventuales efectos sobre las relaciones entre estos medios y el Estado, vivo y presente en la vida política argentina. Asimismo, Yin (2009) insiste en la pertinencia de esta estrategia para responder preguntas de investigación que buscan responder el "cómo" y el "porqué" de un proceso, especialmente cuando el investigador tiene escaso control sobre los eventos y estos ocurren contemporáneamente en la vida real.

En este artículo nos encontramos ante un estudio de caso que combina los tipos descriptivo y explicativo, ya que el trabajo no se limita a describir el fenómeno abordado sino también a explicarlo (Yin, 2009; Wimmer \& Dominick, 2011). Nuestra voluntad es entender un complejo fenómeno social, tratando de retener su máximo significado posible. El caso escogido para conseguirlo es Barricada TV, una televisión comunitaria del barrio de Almagro (en la ciudad de Buenos Aires) con una serie de características específicas que la convierten en un observatorio útil para los objetivos marcados aquí: responde claramente a las características de una televisión comunitaria; sus representantes han batallado constantemente tanto para la aprobación de la ley en 2009 como para su aplicación un vez vigente; su directora, Natalia Vinelli, es a la vez presidente del Espacio Autónomo de Televisoras Comunitarias, Autónomas y Populares (EATCAP) - entidad que se ha enfrentado al gobierno de manera constante en el período 20092015- y finalmente, Barricada TV se vio claramente beneficiada cuando el Estado se decidió aplicar la LSCA en los aspectos relativos a los medios comunitarios. No obstante, hasta setiembre de 2016 continúa sin poder transmitir por abierto ya que el espacio asignado se encuentra interferido por la señal de Canal 13 (del Grupo Clarín). Esta situación ha sido denunciada en reiteradas reuniones al ENaCom, que no brinda respuestas satisfactorias a la organización. A nuestro juicio, este caso permite abordar las intensas relaciones entre el fenómeno estudiado y el contexto ya señaladas como importantes para esta investigación.

Una de las virtudes del análisis de caso es que se trata de una estrategia híbrida, que permite la triangulación de diferentes técnicas de investigación (Yin, 2009; Wimmer \& Dominick, 2011; Venesson, 2013). La más común es la entrevista semiestructurada, 
una técnica especialmente adecuada para captar valores e interpretaciones que ayuden a entender la situación estudiada y el significado que le atribuyen sus protagonistas (Soriano, 2007). En esta investigación se ha usado para describir e interpretar la evolución en el tiempo de Barricada TV, especialmente su relación con el Estado. Se ha combinado con la investigación empírica secundaria (Soriano, 2007) —centrada en las subvenciones a medios comunitarios por parte del Estado- y el análisis documental, tanto de investigaciones científicas previas como de documentación oficial, especialmente leyes, decretos y órdenes. La combinación de estas técnicas enmarcadas en la estrategia del análisis de caso nos ha permitido recoger numerosos indicios y evidencias que sustentan los resultados recogidos en este artículo.

\section{La LSCA: breve repaso por su proceso de debate, sanción y aplicaciones}

La LSCA nació luego de un proceso complejo, definida por un fuerte debate político-mediático y la judicialización de la norma por parte de algunos de los actores más afectados. Esto provocó que, durante el periodo 2009-2015, su aplicación haya sido parcial y, en lo referente a la televisión sin ánimo de lucro, efectivamente menor.

Ya la gestación de la norma fue muy singular. En 2008, momento en que el gobierno de Cristina Fernández de Kirchner comenzó un enfrentamiento significativo con algunos de los grupos multimedia más potentes del país - especialmente el Grupo Clarín-, el Poder Ejecutivo Nacional decidió impulsar una ley que derogara la norma de facto sancionada durante la última dictadura (en 1980) y cuyas reformas - durante la década 1989-2001 (Marino, 2007) - permitieron una fuerte concentración del sistema mediático en pocas manos (Mastrini, 2009; Marino, Mastrini \& Becerra, 2011). Para la elaboración de la nueva ley el Gobierno se inspiró, entre otros aspectos, en el documento 21 puntos básicos por el derecho a la comunicación propuesto por la Coalición para una Radiodifusión Democrática que desde 2004 reclamaba la desconcentración y democratización de los medios y atribuía a los medios comunitarios un papel central en este proceso (Busso \& Jaimes, 2011; Guimerà, 2013).

Esa voluntad de legitimar una ley con fuerte incidencia en el sistema mediático llevó al gobierno a un proceso de redacción participativo. Así, el período de debate constituyó un proceso peculiar, dado que se llevó adelante una serie de consultas con la ciudadanía que puede ser comprendida como instancias de participación en el diseño de la política pública. Así, en marzo de 2009 la presidente Kirchner presentó el anteproyecto de ley a la ciudadanía. A partir de allí se llevaron adelante 25 foros en diferentes localidades del país, en los cuales los ciudadanos - en términos individuales o como parte de un colectivoexpresaban su postura respecto del texto. Como resultado, el proyecto de ley presentado al Congreso en agosto de 2009 contemplaba 512 cambios respecto del anteproyecto. Entre las cuestiones abordadas se incluía la definición para los medios gestionados por 
organizaciones sin fines de lucro. Una vez en la Cámara de Diputados, el proyecto también fue discutido de modo abierto en audiencias públicas para que los ciudadanos y agrupaciones incidieran en el debate. Con la media sanción obtenida en setiembre, fue debatido en el Senado, también previa realización de audiencias en ese escenario, y aprobada definitivamente en octubre de 2009 (Becerra, Marino \& Mastrini, 2012).

La aplicación de la LSCA ha sido sesgada, intensa y dinámica, y puede ser caracterizada así por varias razones (Marino, 2014). Resultó irregular por responsabilidad del Gobierno, que se concentró en algunos aspectos y olvidó otros. Esto se combinó con la reacción de los grupos privados concentrados, que al verse afectados por la norma llevaron adelante acciones que derivaron en un proceso de judicialización que afectó a su desarrollo.

En lo que refiere al gobierno, este se centró en aplicar los aspectos de la ley que buscaban la reducción del peso del Grupo Clarín dentro del sector y dejó en un segundo plano a los medios sin fines de lucro, uno de los teóricos elementos centrales de la ley (Marino, 2014; Di Santi \& Riera, 2016). El plan técnico de frecuencias, que habría de concretar la reserva de un tercio del espectro para los medios no lucrativos y hacer posible la concesión de frecuencias también allí donde el aire estaba saturado por emisores comerciales, sigue pendiente en 2016. Asimismo, se criticó la utilización de los medios públicos - potenciados a partir de las previsiones de la ley-como herramientas gubernamentales y la falta de transparencia de los organismos de control del sector (Lacunza \& Marino, 2013). Por su parte, y como reacción inmediata a la aprobación de la ley, el Grupo Clarín realizó una presentación judicial en que denunció la inconstitucionalidad de los artículos 41, 45, 48 y 161 de la LSCA, referentes a los límites a la concentración, propiedad cruzada y topes de mercado, como así también a los mecanismos de adecuación que se establecieron para los grupos que superaran los topes al momento de la sanción (Di Santi \& Riera, 2016). El proceso comprendido como "judicialización" fue la estrategia utilizada por el grupo para demorar la aplicación de la norma, mientras esperaba un cambio de gobierno que pudiera incluir la derogación de la LSCA (Marino, 2016). En los casi cuatro años que duró esa instancia muchos fueron los momentos supuestamente definitivos que luego no resultaron tales. En esa línea se destacan las medidas cautelares. Una de ellas proponía la fecha del 7 de diciembre de 2012 como tope para el momento a partir del cual los medios excedidos en la cantidad de licencias se adecuaran al nuevo límite, al menos presentando un "plan de adecuación" que debía ser aprobado por la AFSCA.

Eso derivó en una campaña discursiva potente, denominada "7D" (por 7 de diciembre), en la cual el gobierno buscaba plantearse como el defensor de la democracia (comunicacional y en sentido amplio) en un enfrentamiento contra las "corporaciones" mediáticas. Pero la fecha fue pospuesta en sede judicial. El recorrido duró hasta 
octubre de 2013, cuando la Corte Suprema de Justicia de la Nación (CSJN) —-máxima autoridad judicial del país- dictaminó y cerró las posibilidades de discutir en sede judicial, dándole la razón a la postura del gobierno. La LSCA fue declarada constitucional en todos sus aspectos en una decisión que estableció un fallo sólido y argumentado. En su texto, los jueces dieron cauce a demandas vinculadas con la génesis de los famosos 21 puntos en los que se basó gran parte de la ley.

El derrotero político en la Autoridad Federal de Servicios de Comunicación Audiovisual (AFSCA), atribuible al gobierno, tampoco ayudó. Como organismo de aplicación de la ley, el ente sufrió los avatares del período y afectó a una de sus principales funciones: crear institucionalidad en la gestión de políticas de medios en un país que no se ha caracterizado por este elemento en esa materia (Becerra, 2010). De este modo demoró muchos meses en funcionar del modo abierto y participativo. Hasta julio de 2010 estuvo inhabilitada por las medidas cautelares que impidieron la aplicación integral de la ley. Desde su creación funcionó como un espacio de poder del gobierno, que nombró una seguidilla de directores que impidió una acción a largo plazo, con cambios decididos a partir de avatares de la dinámica política. La oposición parlamentaria tuvo dos estrategias que también alteraron su actividad. Primero, no designar entre 2009 y 2011 a sus representantes para deslegitimar la LSCA. A partir de 2012, disputar y reclamar desde la institución tras nombrarlos, aunque uno fue impugnado, lo que retardó el normal funcionamiento hasta 2013. La designación en octubre de 2012 del diputado Martín Sabatella como director levantó el perfil del ente, se convocó al nombramiento de representantes de la oposición y abrió la convocatoria de los planes de adecuación (Becerra, 2016a; Marino, 2014).

La dinámica y el enfrentamiento fueron los rasgos característicos de un período que a principios de 2016 parece haber concluido. Además, la actitud del nuevo gobierno de Mauricio Macri ante los medios y la LSCA abre nuevas dudas sobre una ley todavía con muchos elementos pendientes de aplicación (Becerra, 2016b). Y ahora que el futuro llegó, opera la incertidumbre.

\section{Los medios sin fines de lucro en la LSCA}

Como sostienen diversas investigaciones (Marino, 2009; Marino, Mastrini \& Becerra, 2011; Guimerà, 2013), uno de los aspectos más novedosos de la LSCA es la centralidad que otorga a los medios sin fines de lucro dentro del sistema audiovisual argentino.

Por una parte, el artículo 2 los reconoce como uno los tres actores con derecho a operar servicios audiovisuales, junto con los de gestión estatal y los de gestión privada. Es más, el reconocimiento a los tres tipos se justifica por ser la mejor manera que tiene el Estado de "salvaguardar" los derechos a la información y a la participación de 
la ciudadanía, así como la libertad de expresión. Para hacer posible esto, el artículo 89 reserva el 33\% "de las localizaciones radioeléctricas planificadas, en todas las bandas de radiodifusión sonora y de televisión terrestres, en todas las áreas de cobertura para personas de existencia ideal sin fines de lucro. Las reservas de frecuencias establecidas en el presente artículo no pueden ser dejadas sin efecto". Hay pocos lugares del mundo donde se haya reservado tanto espacio a este sector. A su vez, el artículo 49 prevé la concesión de éter sin concurso previo a emisoras "de muy baja potencia" en zonas de "alta vulnerabilidad social y/o de escasa densidad demográfica" siempre que se destinen "a satisfacer demandas comunicacionales de carácter social". Esta posibilidad es excepcional y siempre que haya frecuencias disponibles.

La ley también establece una serie de garantías y de excepciones para hacer posible un tercer sector viable. Así, los pliegos de los concursos de concesiones de frecuencias deberán ser elaborados "teniendo en cuenta características diferenciadas" según se trate de adjudicaciones para medios con o sin afán de lucro (art. 33). Asimismo, excluye a las entidades sin ánimo de lucro de la prohibición de ser titulares de licencias de servicios de comunicación audiovisual aun siendo prestadores por licencia, concesión o permiso de un servicio público nacional, provincial o municipal (art. 30, excepción del art. 25, inciso d).

Finalmente, el artículo 97 fija que los "servicios de comunicación audiovisual comunitarios, de frontera y de los pueblos originarios” podrán ser receptores de ayudas económicas del Estado equivalentes a un 10\% de los gravámenes pagados por los concesionarios comerciales.

Si bien es cierto que la LSCA promueve la consolidación de un sector no lucrativo importante, no deja claro a qué se refiere. Se reconoce a "prestadores privados sin afán de lucro", pero no se los define a pesar que el artículo 4 está dedicado precisamente a concretar conceptos clave de la ley. Sí define, en cambio, "emisoras comunitarias", que entiende como "actores privados que tienen una finalidad social y se caracterizan por ser gestionadas por organizaciones sociales de diverso tipo sin fines de lucro. Su característica fundamental es la participación de la comunidad tanto en la propiedad del medio, como en la programación, administración, operación, financiamiento y evaluación. Se trata de medios independientes y no gubernamentales. En ningún caso se la entenderá como un servicio de cobertura geográfica restringida". Es decir, unos medios que van más allá de la ausencia de lucro.

Solo posteriormente, a través del Decreto 1225/2010 de desarrollo de la ley, se estableció que son entidades sin afán de lucro "las cooperativas, mutuales, fundaciones y asociaciones definidas como tales por las normas vigentes". Así pues, se citan formas de propiedad pero no hace ninguna referencia a su carácter participativo. La combinación de ambas definiciones ha dado lugar a lo que Torres (2011, p. 59) define como un "pa- 
radoja": mientras la ley apela a la necesidad de medios comunitarios que estimulen la participación ciudadana, el marco legal acaba amparando bajo el concepto de medios sin afán de lucro "un universo muy amplio que excede lo comunitario", por lo que los fundamentos legales de LSCA "quedan desnaturalizados".

Esta relativa ambigüedad conceptual no es casual y nace de la forma como se organizó el proceso de elaboración de la ley. En la discusión sobre su redacción participaron las organizaciones del sector, que no coincidían en qué definición era más útil. Así, algunos sectores minoritarios del movimiento de los medios comunitarios y populares pidieron que se los reconociera explícitamente dentro del articulado y se los regulara aprovechando la definición del artículo 4 (Vinelli, 2011, p. 89). Su intención era defender unos medios débiles y también frenar la entrada de otros actores (sindicatos, iglesias, fundaciones) que pudieran apelar a su naturaleza no lucrativa para tomar posiciones. Otras entidades de mayor densidad, en cambio, se mostraron favorables a una definición lo más genérica posible para evitar la exclusión de medios no lucrativos alejados de planteamientos comunitarios (Guimerà, 2013). Como reconoce Vinelli, la versión aprobada de la ley fue un compromiso entre esa concepción más amplia del tercer sector (que da cabida a fundaciones como la Noble, Ford o Telefónica) y un reconocimiento específico a los medios comunitarios, fruto de sus demandas. En palabras de Vinelli (2015): "[los medios comunitarios] entendíamos que era clave tener voz en ese debate, porque no tenerlo te dejaba hablando en el vacío respecto de lo que habían sido 30 años de demanda por la democratización del sistema”. El resultado fue una delimitación muy amplia con voluntad inclusiva pero que - cómo veremos- no resultó neutra a la hora de aplicar la ley.

\section{La relación Estado-medios sin fines de lucro en la Argentina (1983-2013)}

La aprobación de la LSCA supuso un hito para la radiotelevisión argentina sin afán de lucro, ya que cambió la relación de estos medios con el Estado después de décadas de prohibiciones y persecuciones. El Decreto-Ley 22285/80, aprobado en 1980 por la dictadura pero que rigió hasta 2009, excluía a los agentes sin afanes lucrativos del acceso al espacio radioeléctrico, ya que el artículo 45 solo permitía que fuera explotado por actores privados comerciales. La ley atendía a los intereses de los empresarios afines a los militares e instalados sobre todo en Buenos Aires, dando lugar a un sistema "centralista y privatista, con muchos sectores excluidos de sus derechos" (Postolski \& Marino, 2009, p. 171; Marino, 2009, p. 59).

Si bien es cierto que la norma sufrió diversos cambios, la mayoría reforzó ese carácter. Solo con la aprobación de la Ley 26.053 en 2005 se produjeron cambios que facilitaron el acceso de los actores sin afán de lucro a las ondas aunque en condiciones poco estables a través de los llamados "permisos precarios provisorios". Partiendo de 
esta base legal, el Comité Federal de Radiodifusión (COMFER) — antecedente de la AFSCA - reconoció el funcionamiento y la titularidad de 126 estaciones de radiodifusión sonora pertenecientes a distintas asociaciones civiles sin fines de lucro mediante la Resolución 753/06. Este proceso tuvo cuatro características: solo legalizó emisoras de radio; básicamente reconoció a radios en funcionamiento; era provisional y obligaba a presentarse a un concurso de concesiones en el futuro, y resultó complejo para algunos actores que no consiguieron superarlo. Todo ello lo dota de un carácter claramente discriminatorio y precarizador (Guimerà, 2013).

La LSCA venía a poner fin a esta situación, pero a pesar de la ola de optimismo que provocó su aprobación en 2009, hubo que esperar años para que las primeras televisiones comunitarias lograran en 2013 un mínimo reconocimiento precario similar al de las radios en 2006 y a que se convocaran concursos para obtener plena regulación legal. Mientras tanto, el Estado mostraba señales equívocas sobre su voluntad respecto de los medios comunitarios, poniendo en duda su (supuesta) intención de impulsarlos de acuerdo con la ley.

Así, la AFSCA convocó dos concursos en 2011. El primero era para la asignación de 222 canales de TDT (112 de ellos para entidades no lucrativas), de potencias variables y la mayoría ubicados en capitales y grandes ciudades. Después de un largo periplo, el concurso acabó suspendido en julio de 2012 entre fuertes críticas por los costes económicos que imponía a los concesionarios y por haber fijado un pliego de cláusulas que no diferenciaba entre actores comerciales y no comerciales, tal como mandaba la LSCA. Muchas entidades no lucrativas decidieron no presentarse al concurso por considerar que las excluía (Guimerà, 2013). Mientras este se desarrollaba, la AFSCA convocó un segundo para estaciones de baja potencia y con la voluntad, en principio, de regular las emisoras comunitarias en funcionamiento. Pero salvo las condiciones económicas (que nunca se fijaron), el resto eran las mismas del concurso anterior. Además, no se garantizaba la regulación de todas las televisiones existentes. Este concurso también se suspendió en julio de 2012.

La justificación oficial tanto del gobierno como de la AFSCA fue, precisamente, la necesidad de reelaborar los pliegos para facilitar el acceso a los medios no lucrativos. El entonces director de AFSCA, Santiago Aragón, reconocía que el concurso acabó siendo una prueba para evitar futuros errores, aceptando de hecho que no habían respondido a las necesidades de los no comerciales (Guimerà, 2013). Aragón afirmaba que para finales de 2012 se iban a convocar dos nuevos concursos: uno para organismos sin afán de lucro más dotados económicamente y otro para televisiones comunitarias, populares y alternativas. Un concurso de estas características no se convocó hasta febrero de 2015 y se resolvió en noviembre de 2015, pocas semanas antes del cambio de gobierno que modificaría en profundidad la LSCA. 
Guimerà (2013) demuestra que el comportamiento del Estado hasta ese momento se debe a una mezcla de falta de interés por promocionar estos actores y de una incomprensión notable de la naturaleza del sector audiovisual, en especial, de los medios no lucrativos. Los elementos que apuntan en esa dirección son múltiples. En primer lugar, el hecho que los pliegos de los concursos resultaran excluyentes para los medios no lucrativos a pesar de las advertencias de los representantes del sector. Parece claro que el Estado no entendía su verdadera naturaleza y, en consecuencia, no los trataba de acuerdo con sus características y necesidades. En segundo lugar, el hecho que los agentes gubernamentales no vieran en esos medios un actor útil en su batalla por democratizar la comunicación y, sobre todo, capaz de competir con los grandes grupos a los que el ejecutivo estaba enfrentado. El objetivo prioritario era, y así se reconocía explícitamente desde AFSCA (Guimerà, 2013), desconcentrar a Clarín. Así, con los concursos no se buscaba promocionar a televisiones no lucrativas sino impulsar nuevos medios comerciales que pudieran robarle cuota de mercado; una clara perversión del espíritu y la letra de la ley. Además, los concursos se convocaron sin estar elaborado el plan técnico de frecuencias que obliga la ley, de manera que no se podía saber cuál era el espacio radioeléctrico disponible y a qué corresponde el 33\%. Finalmente, la ausencia de un plan de apoyo económico y de digitalización pensado para estos actores acabó excluyéndolos aún más, a pesar de ser las entidades más débiles (Becerra, Marino \& Mastrini, 2012; Guimerà, 2013). Como sostiene Segura (2013, p. 182), el menor poder relativo de estos medios frente a las grandes empresas privadas y el Estado ayuda a explicar sus problemas para introducir sus necesidades en la agenda política.

Como resultado de todo ello, la percepción del sector sin fines de lucro a mediados de 2012 era que, visto este comportamiento de los estamentos políticos, se debería volver a luchar - como ya se había hecho por la aprobación de la ley- para que se aplicasen las previsiones que permitieran el acceso a las transmisiones por parte de las televisoras (Guimerà, 2013). Como veremos a continuación, estas previsiones se cumplieron para parte del sector. En concreto, para los medios comunitarios.

\section{Las relaciones Estado-medios sin fines de lucro tras el reconocimiento (2013-2015)}

Las televisiones comunitarias tuvieron que esperar hasta 2013 para percibir que su situación había cambiado a pesar de acumular ya cuatro años de vigencia de la LSCA. Ahora bien, la situación no se normalizaría de acuerdo con la ley hasta finales de 2015, cuando se convocaron y resolvieron concursos de asignación de frecuencias. En un contexto de duro combate jurídico con los grandes medios que no se resolvió hasta octubre de 2013 y una AFSCA que era escenario de otro combate - ahora político-, las televisiones no lucrativas quedaron relegadas en las políticas de comunicación argentinas. 
Es en ese marco que la AFSCA emitió una serie de "autorizaciones precarias provisorias" (APP). Esta medida no resulta de un decreto del Poder Ejecutivo ni una acción adoptada por el Congreso Nacional. Como ya se ha señalado en el caso de la radio en 2006, se trata de una decisión de la autoridad de aplicación, un mecanismo habitual en las políticas de comunicación de la Argentina. Es un tipo de autorización que funciona como una especie de paliativo que implica un reconocimiento formal para este tipo de medios, que operan en la incertidumbre limitada por el reconocimiento de su derecho y la falta de política integral para el otorgamiento de licencias y autorizaciones regulares. Así, el Estado reconoce la existencia de esas emisoras y toma nota de que están ocupando un determinado punto en espacio radioeléctrico.

Según Becerra (2014), las autorizaciones provisorias persiguen varios objetivos, entre los que destacan dos. Por una parte, en un marco en el cual el Estado no abría concursos de frecuencias para medios sin afán de lucro, dar cierta condición de "reconocimiento" ante la posibilidad de que un cambio de gobierno implicara un cambio en el paradigma que reconoce la existencia de medios sin fines de lucro, como pareció suceder durante 2016. Por la otra, el objetivo es habilitar a las emisoras a postularse a los concursos en el marco del Fondo de Fomento Concursable para Medios de Comunicación Audiovisual (FOMECA), destinado a proyectos especiales de comunicación audiovisual y apoyo a servicios de comunicación audiovisual, comunitarios, de frontera y de los pueblos originarios.

Pero para Vinelli (2016), las APP evidenciaron la incapacidad de la AFSCA para convocar a concursos que responderán a las necesidades y realidades particulares de este tipo de medios, como quedó demostrado en los procesos suspendidos en 2012. Así, el Estado avanzó con una serie de autorizaciones precarias a canales de televisión analógica de baja potencia; entre ellos, Barricada TV y Canal 5 de Almagro. De esta manera, y a pesar de todo lo que implica la LSCA, el gobierno de Cristina Fernández de Kirchner resolvió otorgar vía AFSCA el mismo tipo de permisos precarios y provisionales que había otorgado a 126 radios en 2006. Ahora bien en el caso de la TV sin fin de lucro, el número es sensiblemente menor. Así, a partir de la Resolución 1273/13 de la AFSCA, primero se otorgaron permisos a 20 canales en octubre de 2013, de los cuales en su mayoría eran pequeños medios comerciales. Luego, a principios de 2014, se sumaron 18 canales más aprobados por las resoluciones 50/2015 (13 canales) y 202/2015 (5 canales). En total, sumaron 38 los canales de baja potencia autorizados.

Incluso dentro de este proceso de reconocimientos, los medios comunitarios fueron relegados y tuvieron que luchar para ser aceptados. En la primera tanda, solo dos medios autorizados estaban gestionados por asociaciones y otros dos, por cooperativas de trabajadores. El resto eran pequeñas empresas audiovisuales. En la 
segunda resolución se aprobó otro canal gestionado por una asociación (Barricada TV) y en la tercera tanda se reconocieron tres más (dos asociaciones y una cooperativa). Como resultado, solo 8 de las 38 autorizaciones corresponden a medios de corte comunitario. Además, cabe tener en cuenta que la segunda y tercera tandas se dieron después de protestas. El Espacio Autónomo de Televisoras Comunitarias, Autónomas y Populares (EATCAP) organizó manifestaciones frente a la sede de la AFSCA y solo después se abrió la puerta a más permisos. Vinelli, portavoz del EATCAP, sostiene que hasta bien entrado 2013 no les atendieron en la AFSCA, lo que se interpretaba como un rechazo hacia el sector. Solo después de "una relación muy conflictiva" se consiguió una reunión a partir de la cual lucharon fuerte para "instalarse en el campo".

Para Vinelli, en 2013 "se notaba que [en la AFSCA] estaban buscando cómo resolver una cuestión que no era prioridad, dado que el problema con Clarín era lo principal. Se notaba que no tenía una política para esto". En ese momento, los medios comunitarios tenían cuatro demandas: "la más importante era la legalización", a la que se sumaba "una política de fomento para el sector y un tratamiento diferenciado de los medios comunitarios dentro de los sin fin de lucro y una señal nacional para todos" que gestionarían conjuntamente. Fue entonces cuando se autorizaron los primeros 20 canales, de los cuales solo cuatro eran no comerciales. Ante esto el EATCAP se plantó y optó por las manifestaciones callejeras frente a la autoridad:

$\mathrm{Y}$ en ese momento volvimos a la calle, a todo o nada. Tuvimos el reflejo de salir a la calle enseguida, hacer la marcha más grande todas las que hicimos. Y a partir de ello se destrabó la cuestión, pero en el marco de un conflicto. Llevamos unas 600 personas a las puertas de AFSCA, con apoyo de diferentes organizaciones, sindicatos, etc. Ahí obtuvimos la primera promesa de auditorías a nuestros canales e ingreso a la lista de autorizaciones. A partir de ahí desarrollamos una serie de reuniones en las que ellos planteaban una serie de requisitos y nosotros marcábamos que éramos los únicos que teníamos todo en regla (Vinelli, 2015).

Es justo en ese momento cuando se puede observar un primer cambio en la relación entre medios comunitarios y el Estado: se estableció un proceso de negociación en el cual se fueron fijando los criterios que debían cumplir los canales autorizados, de manera que se establecían unos mínimos al mismo tiempo que criterios claros: "tener la cuestión impositiva resuelta, haber sido censados en tiempo y forma y estar emitiendo". Ahora bien, Vinelli sostiene que "había cuestiones políticas: si no teníamos todo en regla no nos autorizarían, mientras que a otros más cercanos sí. Siempre tuvimos esa claridad", ya que desde el poder ejecutivo se percibía a algunos de estos medios como ideológicamente hostiles. Una vez obtenida la APP, Vinelli es clara al afirmar que la relación es "estable" y "de diálogo" con la AFSCA; que les reconoce demandas específicas y afirma ser consciente de la deuda pendiente en la aplicación de la ley. "Estamos en una situación que no es conflictiva, sino de diálogo con el 
organismo, y a sabiendas de que ellos son el Estado", sostiene Vinelli (2016) ante lo que es un evidente giro de 180 grados en la valoración de la relación con el Estado.

Esta relativa normalidad se ratificaría a lo largo de 2015 cuando la AFSCA convocó en mayo y resolvió en diciembre un concurso de frecuencias de televisión digital terrestre (conocida en la Argentina como televisión digital abierta [TDA]). De acuerdo con el pliego hecho público a través de la resolución 39/2015, por primera vez en la historia del país se convocó un concurso donde se fijaban condiciones diferentes para operadores con fin de lucro y sin él. Para el director de la AFSCA, Martín Sabbatella, se trataba de "otro gran paso en la aplicación de la Ley de Servicios de Comunicación Audiovisual, que permitirá seguir democratizando la televisión en la Argentina [...] y ampliar la oferta de señales, hacerla más plural y diversa, y aumentar la generación de contenidos audiovisuales y las fuentes de trabajo en el sector" (Lacunza \& Marino, 2013).

En total se convocaron 21 frecuencias de alta potencia (nueve para entes sin ánimo de lucro y doce para comerciales) y 60 de baja potencia repartidas por ocho zonas del país, a la que se podían presentar los dos tipos de actores. En este sentido, el pliego para las emisoras de baja potencia no comerciales era más accesible que los formulados en los concursos de 2012. Así, el coste del pliego era un 75\% menor para las entidades no lucrativas, la documentación a presentar era más simple y las certificaciones a presentar, menores; las propuestas técnica y de inversiones habían sido simplificadas; y las obligaciones adquiridas ante las entidades gestoras de derecho eran menores. A pesar de ello, Vinelli sostiene que fueron "unos pliegos difíciles pero realizables", que a pesar de ser más baratos siguieron siendo caros para medios con escasos recursos, tanto materiales como económicos y humanos. Esto hizo que pocos medios con este perfil se presentaran al concurso y que solo tres obtuvieran concesión: Pares TV, Urbana TV y Barricada TV. En total, apenas un 4\% de las frecuencias concursables. La propia Natalia Vinelli (2016) reconoce que AFSCA facilitó en esa ocasión las gestiones hasta el punto que ofreció cursos de capacitación y asesoramiento para completar las solicitudes. Para esta representante del sector, "los concursos son un escalón fundamental en la pelea por la legalización, logrados tras un largo período marcado por la movilización y el reclamo" (Vinelli, 2016, p. 57).

Como señalan Becerra et al. (2016), es posible inferir que la política de reconocimientos al sector no lucrativo se orientó a la televisión por cable en desmedro de la radio FM y AM e incluso de la televisión abierta. Esto en parte puede explicarse porque el proceso de adjudicación de licencias de cable no requiere concurso, como es el caso de los otros medios. También por la posibilidad de desarrollo de las cooperativas de cable preexistentes. En cualquier caso la situación expresa el débil lugar que le toca a la televisión abierta sin afán de lucro. 


\section{Análisis de caso: Barricada TV}

Barricada TV es, por múltiples y diversas razones, un caso singular dentro de las televisiones sin fines de lucro argentinas. Ubicada en la ciudad de Buenos Aires, está gestionada por la Asociación Civil Trabajo, Educación y Cultura y se inserta en un espacio físico peculiar: la fábrica recuperada IMPA, con amplio recorrido en la militancia de base y cuyos trabajadores lograron hacerse con la propiedad en 1998 después de su quiebra en el contexto de la fuerte crisis económica argentina de finales de los años noventa.

Como define su directora, Natalia Vinelli (2015), la formación de Barricada TV no fue individual sino que fue un proceso colectivo. Y ese es el modo en que comprenden el desarrollo del medio y su gestión. Y no (se) puede despegar el derrotero de una organización sin el de aquella que la contiene. "Empezamos a pensar en la necesidad de instalar el canal a través de un acuerdo político y de afinidad importante con la fábrica recuperada IMPA, la primera empresa recuperada que es una metalúrgica que logró su ley de expropiación", afirma Vinelli (2015). Esto le confiere una identidad particular, ya que el canal nace como parte del movimiento piquetero, "que ya había desarrollado una política de movilización, vinculación, pelea por subsidios, planes, vuelta al conflicto para reclamar mínimos derechos, siempre en la línea del trabajo digno y genuino, pero en el marco de la búsqueda de organización para la subsistencia”.

Como sostiene Vinelli, esto también marcó la relación (posible) de la televisión con la administración pública: "Nosotros criticamos un conjunto de cuestiones del Estado como aparato burocrático del gobierno. Pero entre la corporación mercado y la corporación Estado nos quedamos toda la vida con la corporación Estado". En esta línea, ella hace una lectura positiva de la experiencia de Barricada dentro de IMPA: "la actitud batalladora nos dio gimnasia para la relación con el Estado". De hecho, Barricada TV es un ejemplo ilustrativo de todas las fases que han estado atravesando las emisoras comunitarias tanto en lo referido a su modelo de gestión como también a su relación con el Estado en general y la AFSCA en particular.

Ya en el proceso de elaboración de la ley audiovisual, Barricada TV, a través de su directora Natalia Vinelli (2015), insistió en la principal demanda del medio y del conjunto de medios comunitarios: la legalización. Ahora bien, la entendían como un punto de partida de un proceso que debía afrontar la concentración mediática mediante la modificación de la regulación, la implementación de una política pública y la ampliación de las voces populares. "Queremos mostrar lo que los medios no muestran, desde nuestros objetivos, que son políticos. Para ello tenemos que llegar a todo el mundo", sostiene Vinelli. Desde este planteamiento, cuando se discutió la ley y se debatió el proyecto, su línea fue un acuerdo general con otros actores favorables a la nueva regulación y al mismo tiempo marcaba sus diferencias desde una concepción política: 
cuestiones vinculadas con el tratamiento diferenciado, la definición de medio comunitario, los pliegos específicos. De hecho, Vinelli fue como representante de EATCAP una de las voces más críticas con la laxa definición de medios sin fines de lucro y la ausencia de una mayor concreción del modelo comunitario en el redactado de la ley (Guimerà, 2013).

Una vez aprobada la ley, Barricada TV formó parte activa de las movilizaciones para la legalización de los medios populares y la aplicación efectiva de la LSCA, y constituyó el EATCAP junto con experiencias como Faro TV, TVPTS, En Movimiento TV y Canal 4 Darío y Maxi. En este marco denunció los concursos ya citados suspendidos en 2012 por su carácter restrictivo debido a las exigencias establecidas, el costo de los pliegos y el pago de un alto canon a ARSAT para la transmisión de la señal (Resoluciones 685/2012 y 686/2012 de la AFSCA). También realizó una propuesta con varias modificaciones a las condiciones instauradas mediante un nuevo llamado a concurso convocado a través de la resolución 1465/2012 (Vinelli, 2016).

Todo este recorrido previo convierte a la situación de Barricada TV en la materialización del cambio en las relaciones entre el Estado y el sector sin actividades lucrativas luego de 2013. Barricada TV fue una de las televisiones que obtuvo permiso -después de batallarlo largamente - para emitir de forma provisional en analógico mediante la Autorización 50/14 de AFSCA del 9 de enero de 2014, que otorgó a la Asociación Civil Trabajo, Educación y Cultura la frecuencia 5 de la ciudad de Buenos Aires. En diciembre de 2015, la AFSCA le concedió a través de la Resolución 1092/2015 la concesión para emitir legalmente después de ganar el concurso abierto en mayo y largamente esperado desde 2009 y, en especial, desde 2012.

El complejo proceso de legalización tuvo implicaciones en tres grandes aspectos de la relación con el Estado: cumplimiento de la LSCA, publicidad oficial y subvenciones. En el primer punto, Vinelli señala que la falta de reglamentación de la ley y la poca diferencia entre medios comerciales y no lucrativos fija obligaciones genéricas difíciles de cumplir para los medios más modestos. Cita específicamente las tres horas diarias de programación infantil, las obligaciones en materia de lenguaje de signos, la imposición de emitir un 30\% de producciones locales independientes y la imposibilidad de lotear espacios. Para Vinelli (2015), el sector "no estaba maduro" para darse cuenta de estas implicancias al ser legalizados e insiste en dos cosas: en el problema de origen de la ley de no diferenciar a los medios comunitarios y en la necesidad de reglamentaciones específicas que ajusten la ley a sus características.

La organización reconoce haber dado "una discusión enorme" a la posibilidad de recibir o no publicidad oficial, ya que se percibía como un problema de probable influencia política sobre el medio. También genera problemas el hecho de tener que aceptar publicidad electoral, especialmente la de partidos que el medio no apoyaría nunca. 
Durante un año y medio se debatió si se daban los pasos necesarios para recibir publicidad del Gobierno Ciudad de Buenos Aires. "Tuvimos una posición muy principista en el inicio sobre esto. Luego la revisamos porque nos dimos cuenta de a quién le convenía eso. Todo lo que es publicidad del Estado ahora no nos genera ningún problema. Antes era una cuestión. Sostener el canal demanda fuentes de financiamiento, tiempo y dinero. Y se vuelve complicado". De hecho, Vinelli (2015) afirma que la publicidad es fundamental para poder pagar los salarios de los profesionales que sostienen el medio.

En cambio, los subsidios nunca fueron un problema. El artículo 97.f de la LSCA establece que el $10 \%$ de lo recaudado por la autoridad de aplicación debe destinarse a la promoción de los medios comunitarios, especialmente para ayudarlos en el tránsito hacia la tecnología digital. De acuerdo con esto, en 2013 se crearon los fondos FOMECA y se convocaron los primeros concursos para optar a ellos. Dirigidos a entidades sin fines de lucro y comunidades de pueblos originarios, tuvieron como objetivo fomentar la adquisición de equipamiento técnico, infraestructura física y tecnológica, producción radiofónica y audiovisual, y, por último, incentivar la gestión de medios comunitarios.

Tabla 1. Fondos FOMECAS obtenidos por Barricada TV.

\begin{tabular}{llr}
\hline Año & Tipo de línea de fomento & \multicolumn{1}{c}{ Monto } \\
\hline 2014 & Formato microprograma & $\$ 130.000$ \\
\hline 2014 & Gestión & $\$ 138.500$ \\
\hline 2014 & Informativo diario & $\$ 410.000$ \\
\hline 2015 & Gestión de medios comunitarios & $\$ 295.800$ \\
\hline 2015 & Equipamiento de infraestructura para emisoras de TV - Licenciatario operador & $\$ 1.300 .000$ \\
\hline 2015 & Producción de contenidos audiovisuales para emisoras de TV & $\$ 1.300 .000$ \\
\hline
\end{tabular}

Fuente: elaboración propia con base en datos de FOMECA.

Durante 2013 se desarrollaron las primeras tres líneas de fomento, en 2014 se elevaron a siete y en 2015 fueron nueve. Las líneas de fomento se destinaron a distintos objetivos: el equipamiento de radios y la producción radiofónica y audiovisual; para pueblos originarios de diferente tipo ("Comunicación con identidad"); la novedosa línea 5 para "Gestión de medios comunitarios" que se encargó de solucionar otro tipo de problemas de las emisoras como la profesionalización de algunas áreas administrativas. Más tarde se diferenciaron los premios de producción para emisoras y otros para productoras y se sumaron líneas para la vinculación de emisoras y el desdoblamiento de las líneas de gestión para aquellas organizaciones que resultaron ganadoras en 2014 y las que buscaban su primer premio. 
Este mecanismo fue aprovechado de modo relevante por Barricada TV, que en dos años consecutivos obtuvo seis FOMECAS de diferentes líneas y con montos que van desde poco más de cien mil pesos hasta los que superan el millón. Esto le permitió desarrollar importantes inversiones en equipamiento, como puede observarse en la tabla 1. Los dos primeros premios que figuran en la tabla implican ingresos que superan el $10 \%$ del presupuesto anual del canal. Esto, claro, se sobrepasa holgadamente en la línea destinada a "Informativo diario". Los premios obtenidos para equipamiento y producción en 2015 cubren un presupuesto anual y su destino fue aportar al pago del transmisor y los gastos de su operatividad.

En la línea de mejorar la relación con el Estado, Vinelli reclama unos FOMECA destinados a salarios más abiertos, ya que en la actualidad no están pensados para contratar a personas vinculadas con la asociación que gestiona la emisora, hecho que obliga a contratar a gente externa para obtenerlos pero sin la garantía de que la televisora pueda sostenerlos sin recibir la subvención. Para Vinelli, en cualquier caso, es la publicidad la que debe garantizar las nóminas y entiende que los subsidios son "para cosas puntuales".

A pesar de todo el proceso de regulación, a principios de 2016 Barricada todavía tuvo que luchar para poder ejercer todos sus derechos. En abril se manifestó frente al Ente Nacional de Comunicación (ENACOM, sucesor de la disuelta AFSCA) para pedir al Estado que liberase el canal 33 de la televisión digital terrestre que le fue concedido y que estaba ocupado por la cadena comercial nacional Cadena 13. En esta línea, Vinelli también pide que el Estado obligue a las empresas de cable - la principal plataforma de acceso a la televisión en la Argentina- a incluir a los medios comunitarios en su parrilla de contenidos. Para Barricada TV es fundamental estar presente en todas las plataformas de acceso, ya que, de lo contrario, queda limitada su capacidad de penetración en la audiencia. Especialmente si se tienen en cuenta los problemas para difundir por internet, donde los servicios de streaming de calidad son demasiado caros para este tipo de medios, y los gratuitos son de mala calidad. Barricada TV, pues, sigue luchando por "la legalización efectiva para poder acceder a los cables y para que el Estado te suba a la TDT".

\section{Conclusiones}

La experiencia de Barricada TV, un caso singular en el audiovisual argentino, aporta abundante información sobre cómo se ha aplicado la LSCA en la Argentina durante la última parte de los gobiernos kirchneristas (2009-2015) y cómo ha afectado esa aplicación en las relaciones entre los medios comunitarios y el Estado.

En el primer aspecto, todas las evidencias recogidas durante el trabajo muestran una aplicación tardía de la LSCA en los aspectos relativos a la potenciación de la te- 
levisión no comercial y, en especial, de la comunitaria. Además, se sigue observando esa aplicación irregular y sesgada que investigaciones previas de los autores ya habían demostrado (Marino, 2016; Guimerà, 2013). La tardanza en convocar concursos de concesión de frecuencia (2012, después de tres años de vigencia de la ley), lo problemática de su aplicación y su posterior anulación, el recurso a las conflictivas APP y la convocatoria y resolución de un concurso solo a finales de 2015 y ante la previsión de un cambio en el gobierno, muestran algo claro de una administración que había hecho de los medios no lucrativos un caballo de batalla en la reforma del sector audiovisual argentino. Lo que se presentó como el elemento que podría generar la multiplicidad de voces tan anhelada resultó apenas una posición discursiva que no encontró su materialización sino hasta el final del período, en condiciones que generaron incertidumbre sobre el porvenir. En este sentido, hay indicios que apuntan a que solo una vez encauzado (aparentemente) el conflicto con Grupo Clarín con el fin de la judicialización de la ley en octubre de 2013 se encaró la cuestión del sector no lucrativo. Ciertamente, cuesta mucho identificar el relato político basado en los 21 puntos de la Coalición para una Radiodifusión Democrática en la praxis administrativa de Gobierno y la AFSCA en lo relativo a la televisión.

En el segundo aspecto, la investigación llevada a cabo aporta conocimiento sobre cómo se relacionan esos medios comunitarios con el Estado que los persiguió durante décadas y luego le costó reconocerlos plenamente a pesar del explícito texto de la ley. La información recogida apunta en tres direcciones diferentes - que muestran una compleja relación que aún debe consolidarse-, aunque el cambio en la gestión de gobierno y las primeras medidas de la nueva administración pone en duda que continúen las acciones que protegen al sector sin fines de lucro. Por una parte, y como ya se observó en 2012 (Guimerà, 2013), los medios comunitarios han tenido que luchar insistentemente para conseguir una concesión de uso del espectro que los regule plenamente. Barricada TV militó para que la LSCA reconociera explícitamente a los medios comunitarios; peleó por una reglamentación de la ley que los reconozca y los proteja mejor, tuvo que volver al conflicto para obtener una APP que no obtuvo en primera instancia y batalló por la convocatoria de concursos que trataran diferente a medios comerciales y no comerciales. Aún en 2016 tiene que enfrentarse al Estado para estar presente en la televisión digital abierta y que se considere su presencia y la de otros medios comunitarios en la grilla de las cableras. De nuevo, pues, se observa un desencaje entre el texto de la ley y su aplicación por parte de la administración: todo se tuvo que pelear.

La segunda dirección en la que cambian las relaciones con el Estado es la relativa a la propia aplicación de la LSCA. De repente, Barricada TV deja de estar fuera de la ley y tiene que adaptarse a sus obligaciones, que en la normativa genérica no distingue 
- de nuevo- entre medios lucrativos y no lucrativos. Obligaciones de producción y emisión y limitaciones a prácticas habituales del sector (en tanto que fuente de financiamiento) como el loteado de espacios complican no solo la aplicación de un modelo comunicativo diferente sino incluso la propia viabilidad económica de estas televisiones, históricamente frágiles. La tercera dirección de cambio apunta hacia un lado diferente: el acceso a los FOMECA no solo ha permitido acceder a ingresos que han permitido desarrollar proyectos sino que han llegado hasta a triplicar el presupuesto anual de Barricada. Sin duda, esto ayuda a la consolidación de estos medios y a llevar a cabo inversiones estructurales que aportan beneficios a largo plazo. El acceso a la pauta oficial - enfocada específicamente en la del Gobierno de la Ciudad Autónoma de Buenos Aires - completa esta dirección de cambios, aportando unos ingresos que han permitido consolidar equipos humanos, un aspecto que aún sufre limitaciones en 2016.

La aprobación de la LSCA ha abierto la puerta a unas relaciones entre Estado y las televisoras comunitarias mucho más compleja y poliédrica que el histórico enfrentamiento directo. Se pasó de la tradición del Estado/policía que reprimía, confiscaba equipos y limitaba el derecho a la información a los actores comerciales a un modelo que regula e interpela mediante el reconocimiento del derecho y la ampliación de las obligaciones, sin contemplar las especificidades del sector. El Estado es ahora un agente que puede contribuir a la estabilidad de Barricada TV pero con el cual se debe negociar para una reglamentación de la ley que suavice obligaciones legales que dificultan notablemente la viabilidad del modelo comunitario. Sin duda, a finales de 2015 el escenario que se entreveía era el de negociación constante desde la seguridad jurídica que da la concesión de frecuencia. Ahora bien, el cambio de gobierno a partir de 2016 y sus primeros pasos hacia el desmantelamiento práctico de aspectos centrales de la ley no es un buen augurio para el futuro de la televisión comunitaria en la Argentina.

\section{Referencias}

Argentina. Ley No 26.522. Servicios de Comunicación Audiovisual. Disponible en http://servicios.infoleg.gob.ar/infolegInternet/anexos/155000-159999/158649/ norma.htm [consultado: mayo de 2016].

Becerra, Martín (2010). Las noticias van al mercado: etapas de la historia de los medios de la Argentina. En: Lugones, Gustavo \& Flores, Jorge (comps). Intérpretes e interpretaciones de la Argentina en el bicentenario (p. 139-165). Bernal: Universidad Nacional de Quilmes.

Becerra, Martín (2016a). De la concentración a la convergencia: política de medios en Argentina y América Latina. Buenos Aires: Paidós. 
Becerra, Martín (2016b). Restauración. Buenos Aires: Quipu: políticas y tecnologías de comunicación [blog]. Disponible en: https://martinbecerra.files.wordpress. com/2016/01/restauracic3b3n-blog-becerra-ene20165.pdf [consultado: mayo de 2016].

Becerra, Martín; Marino, Santiago \& Mastrini, Guillermo (2012). Mapping digital media: Argentina. London: Open Society Foundations (OSF). Disponible en:

https://www.opensocietyfoundations.org/sites/default/files/mapping-digital-mediaargentina-20121107.pdf [consultado: mayo de 2016].

Becerra, Martín et al. (2016). Diagnóstico sobre el acceso del sector sin fines de lucro a medios audiovisuales en la Argentina. En: Segura, Maria Soledad \& Weckesser, Cintia (eds.). Los medios sin fines de lucro entre la Ley Audiovisual y los decretos: estrategias, desafíos y debates en el escenario 2009-2015 (p. 153-164). Córdoba: Editorial de la UNC [Universidad Nacional de Córdoba].

Busso, Néstor \& Jaimes, Diego (comps.) (2011). La cocina de la ley. Buenos Aires: FARCO.

Di Santi, Matías \& Riera, Ariel (2016). “Un mapa poco adecuado”. En: Marino, Santiago (ed.). El audiovisual ampliado: políticas públicas, innovaciones del mercado y tensiones regulatorias en la industria de la televisión argentina frente a la convergencia. Buenos Aires: Ediciones Universidad del Salvador.

Guimerà i Orts, Josep Àngel (2013). Políticas de comunicación, pluralismo y televisión sin afán de lucro: el caso de Argentina". Ámbitos: revista internacional de comunicación, 23. Disponible en:

http://ambitoscomunicacion.com/2013/politicas-de-comunicacion-pluralismo-ytelevision-sin-afan-de-lucro-el-caso-de-argentina/\# [consultado: mayo de 2016].

Lacunza, Sebastián \& Marino, Santiago (2013, nov. 1). Una encrucijada para dos: Clarín y el Gobierno. En: Ámbito financiero, suplemento Viernes.

Marino, Santiago (2009). El caso Argentina. En: Gómez, Gustavo \& Aguerre, Carolina (coords.) Las mordazas invisibles: nuevas y viejas barreras a la diversidad en la radiodifusión (p.55-76).Buenos Aires:Asociación Mundialde Radios ComunitariasAmérica Latina y Caribe (AMARC-ALC). Programa de Legislaciones y Derecho a la Comunicación.

Marino, Santiago (2013). Políticas de comunicación del sector audiovisual: las paradojas de modelos divergentes con resultados congruentes. Los casos de la Televisión por Cable y el Cine en Argentina entre 1989-2007 [tesis]. Buenos Aires: Universidad de Buenos Aires. Facultad de Ciencias Sociales.

Marino, Santiago (2014). Vaivén: desgranar moralejas en la Argentina de la ley audiovisual. Observatorio latinoamericano, 14, p. 79-91. Disponible en: 
http://iealc.sociales.uba.ar/files/2011/06/Observatorio-Latinoamericano-14-medios. pdf [consultado: mayo de 2016].

Marino, Santiago (ed.) (2016). El audiovisual ampliado: políticas públicas, innovaciones del mercado y tensiones regulatorias en la industria de la televisión argentina frente a la convergencia. Buenos Aires: Ediciones Universidad del Salvador.

Marino, Santiago; Mastrini, Guillermo \& Becerra, Martín (2011). El proceso de regulación democrática de la comunicación en Argentina. Derecho a comunicar, 1, p. 1-17.

Mastrini, Guillermo (ed.) (2009). Mucho ruido, pocas leyes: economía y políticas de comunicación en la Argentina (1920-2004). Buenos Aires: La Crujía Ediciones.

Postolski, Glenn \& Marino, Santiago (2009). Relaciones peligrosas: los medios y la dictadura: entre el control, la censura y los negocios. En: Mastrini, Guillermo (ed). Mucho ruido, pocas leyes: economía y políticas de comunicación en la Argentina (1920-2004) (p. 159-188). Buenos Aires: La Crujía Ediciones.

Segura, María Soledad (2013). Contigo o sin ti: medios no lucrativos y Estado desde la Ley 26.522. Austral comunicación, 2 (2), p. 145-185.

Segura, Maria Soledad \& Weckesser, Cintia (2016). Los medios sin fines de lucro entre la Ley Audiovisual y los decretos: estrategias, desafios y debates en el escenario 20092015. Córdoba: Editorial de la UNC [Universidad Nacional de Córdoba].

Soriano, Jaume (2007). L’ofici de comunicòleg: mètodes per investigar la comunicació. Vic: Eumo.

Torres, Javier (2011). Políticas públicas y comunicación comunitaria.En: Comunicación y televisión popular (p. 55-70). Buenos Aires: El Rio Suena.

Venesson, Pascal (2013). Estudios de casos y seguimiento de procesos: teoría y práctica. En: Della Porta, Donatella \& Keating, Michael (eds.). Enfoques y metodologías en las ciencias sociales: un enfoque pluralista (p. 237-254). Madrid: Akal.

Vinelli, Natalia (2011). Siete chicanas sobre la televisión alternativa. En: Vinelli, Natalia (comp.). Comunicación y televisión popular (p. 89-98). Buenos Aires: El Rio Suena. Vinelli, Natalia (2015). Entrevista realizada por los autores de este artículo en junio.

Vinelli, Natalia (2016). Televisión alternativa y Ley 26.522: diario de una presentación a concurso. En Segura, Maria Soledad \& Weckesser, Cintia (eds.). Los medios sin fines de lucro entre la Ley Audiovisual y los decretos: estrategias, desafíos y debates en el escenario 2009-2015 (p. 51-53). Córdoba: Editorial de la UNC [Universidad Nacional de Córdoba].

Wimmer, Roger D. \& Dominick, Joseph R.(2011). Mass media research: an introduction. 9th ed. Boston: Wadsworth.

Yin, Robert K. (2009). Case study research: design and methods. Thousand Oaks: Sage. 